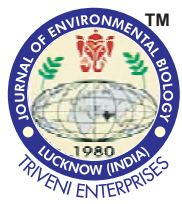

DOI : http://doi.org/10.22438/jeb/39/3/MRN-574

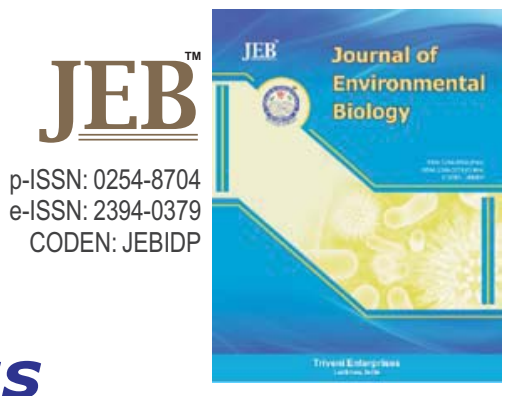

\title{
Interspecies diversity of Bacillus thuringiensis isolates native from North Western Indian Himalayas
}

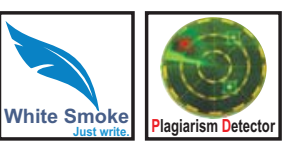

Authors Info

\begin{abstract}
A.R.N.S. Subbanna ${ }^{1,2 *}$, M.S. Khan ${ }^{1}$, R.M. Srivastava', P.K. Mishra ${ }^{2}$, B. Kalyana Babu ${ }^{1}$ and V. Venkateswarlu ${ }^{3}$

1 Department of Entomology, College of Agriculture, Govind Ballabh Pant University of Agriculture and Technology, Pantnagar- 263 145, India

${ }^{2}$ ICAR- Vivekananda Parvatiya Krishi Anusandhan Sansthan, Almora -263 601, India

${ }^{3}$ Department of crop protection, ICAR-Central Tobacco Research Institute, Rajahmundry-533 105, India
\end{abstract}

${ }^{*}$ Corresponding Author Email subbanna.ento@gmail.com

Key words

AP-PCR

Bacillus thuringiensis (Bt)

Genetic diversity

Gyrase B-sequence

Serovars

Publication Info

Paper received : 30.01.2017

Revised received : 22.05.2017

Re-revised received : 16.09.2017

Accepted : 26.09.2017

\section{Abstract}

Aim : Bacillus thuringiensis (Bt) is a ubiquitous pathogenic bacterium exploited worldwide in successful insect pest management programs. The present study aimed at resolving the diversity status and genetic structure of Bt isolates collected from unique and native ecological niche, north-western Indian Himalayas.

Methodology : Forty five Bacillus thuringiensis (Bt) isolates native to Uttarakhand, India were analyzed for their genetic diversity using Arbitrary Primed-Polymerase Chain Reaction (AP-PCR) patterns (ERIC1, ERIC2, BOX and GTG) and gyrase $B$ (gyrB) sequences.

Results : Amongst the AP-PCR patterns, ERIC and BOX primers clearly indicated the genetic inconsistency of $B t$ by differentiating low and high hill isolates with unique banding pattern. A dendrogram generated showed fastidious clustering with coexistence of attitudinally related strains, indicating the genetic relatedness with respect to geographical proximity. The gyrB (285 bp) dependent phylogeny of seven selected isolates revealed their genetic proximity with different serovars.

Interpretation : The present study demonstrates that ERIC and BOX-PCR can be used in establishment of geographical variation and gry $B$ in phylogenetic relatedness with serovars. Genetic relatedness between $B t$ isolates was found to be a function of geographical proximity.

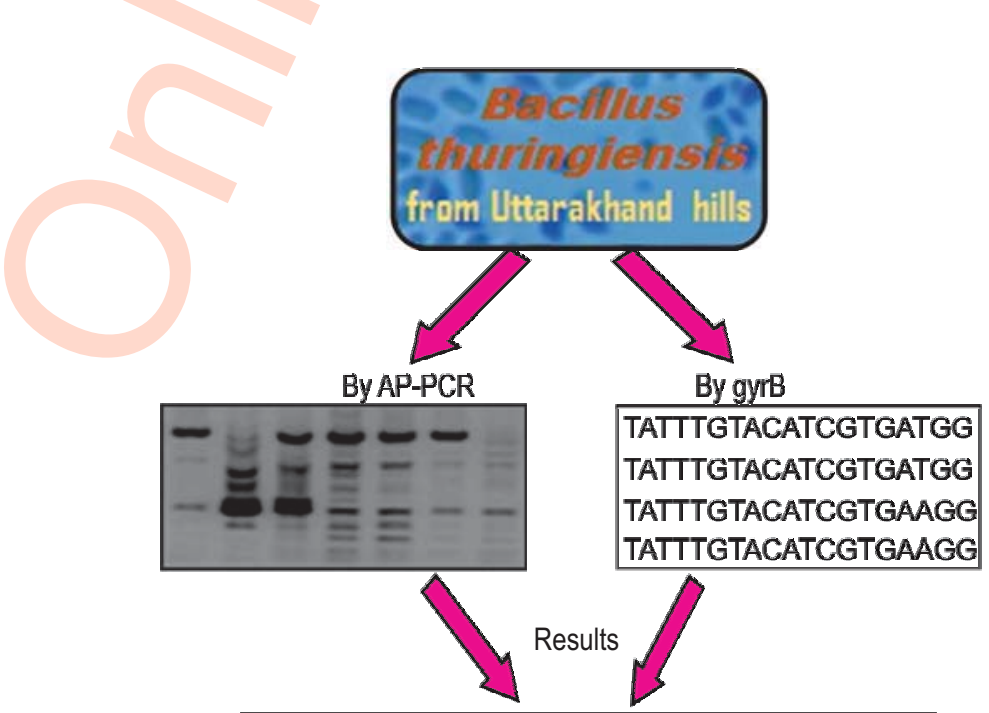

Novel members of family Genetic discrepancy between distant strains Co existence of attitudinally related strains Phylogenetic relatedness with serovars 


\section{Introduction}

Globally rising problems on environmental pollution, resistance development, trade restrictions etc., compels limited use of chemical pesticides against agricultural pests. On the other hand, various pest species belonging to Lepidoptera, Coleoptera and Diptera are continued to be the limiting factors of agricultural production demanding alternative control measures. In this context, use of biopesticides with comparable efficacy to chemical pesticides emerged as a potential tool in successful management of target pests (Chandlera et al., 2008). Amongst the bioinsecticides, Bacillus thuringiensis $(B t)$ occupies prime position in successful materialization as a commercial insect pathogen. The unique mode of action by the bacterium (Sanahuja et al., 2011) offers ecological benefits with no known non-target effects, environmental pollution and bioaccumulation.

Elite strains of $B t$ with toxicity against different non-hosts with putative genes (Kuo and Chak, 1996) having potential in transgenic development has been reported (Kumar et al., 2008). So, it is important to look for new $B t$ strains, and great efforts are presently being undertaken at different research centers (Lima et al., 2002). Polymerase chain reaction árez-Pérez, 2003; Zara et al., 2006), diversity analysis (Awad et al., 2007; Katara et al., 2012) and target prevision (Carozzi et al., 1991) without the need to carry out all the tedious and time-consuming bioassays and biochemical analysis. Regarding the genomic diversity within the $B t$ different molecular approaches have been used, including Restriction Fragment Length Polymorphism (RFLP) (Akhurst et al., 1997), Ribotyping (Joung and Cote, 2001), Random Amplified Polymorphic DNA(RAPD) (Malkawi et al., 1999), Arbitrary Primed Polymerase Chain Reaction (AP-PCR) (Brousseau et al., 1993), Repetitive Extragenic Palindromic sequences (REP-PCR) (Katara et al., 2012). However, direct comparison among the results obtained through these methods complicates assessment of genomic variability (Garcia et al., 2015) of tested Bt collections and were found to be insufficient.

On the other hand, Protein-coding genes exhibit much higher genetic variation, which can be used for classification of closely related taxa (Yamada et al., 1999). The desired features of targeted diversity analysis genes would be that they do not transmit horizontally as plasmid DNA does, their molecular evolution rate is higher than that of $16 \mathrm{~S}$ rRNA and they are distributed universally among bacterial species. Awad et al. (2007) reported that variability in a fragment of $352 \mathrm{bp}$ belonging to DNA gyrase subunit $B$ (gyrB) protein is an efficient, rapid and easy method to make a distinction between native Bt collection. Besides, conservation and understanding diversity in native $B t$ isolates is of prime importance with respect to the evolutionary relatedness and toxicological profiles. Keeping this in view, a collection of $45 \mathrm{Bt}$ isolates native to Uttarakhand Himalayas were studied for their genetic diversity and phylogenetic relatedness using a combination of AP-PCR and gyrB gene sequences.

\section{Materials and Methods}

Bacterial strains : The $B t$ isolates used in this study were collected from different soil samples from all the 13 districts of Uttarakhand state, North Western Indian Himalayas and maintained in pure and viable form in $40 \%$ glycerol stocks at -80 ${ }^{\circ} \mathrm{C}$. The threshold height for mid hills was considered as 1000 to $1700 \mathrm{amsl}$, below and above which were considered as plain/low and high hills, respectively. All the isolates were recovered and retained as single spore colonies in Luria bertani (LB) slants at $4^{\circ} \mathrm{C}$ for ease in use. The bacterial cultures were obtained by growing in LB broth at $250 \mathrm{rpm}$ at $30^{\circ} \mathrm{C}$, unless otherwise specified.

Total DNA extraction : The DNA was isolated by CTAB $\mathrm{NaCl}$ procedure as described by Sambrook and Russel (2001), with some modifications. Briefly, the vegetative cells from an overnight grown culture $(4 \mathrm{ml})$ were pelleted by centrifugation (10000 rpm for $2 \mathrm{~min}$ at $\left.4^{\circ} \mathrm{C}\right)$, washed twice with TE buffer $(10 \mathrm{mM}$ tris- $\mathrm{HCl}, 1$ mM EDTA, pH 8.0) and resuspended in $550 \mu \mathrm{l}$ of same buffer containing $10 \%$ SDS and $1 \mathrm{mg} \mathrm{m}^{-1}$ of proteinase K. After incubation for $1 \mathrm{hr}$ at $37^{\circ} \mathrm{C}$, the cells were lysed by adding $100 \mu$ of $5 \mathrm{M} \mathrm{NaCl}$ and $80 \mu \mathrm{l}$ of CTAB-NaCl solution $(10 \mathrm{mM}$ tris, $20 \mathrm{mM}$ EDTA, $1.4 \mathrm{M} \mathrm{NaCl}, 0.2 \% \beta$-mercaptoethanol in $2 \%$ CTAB solution $\mathrm{pH}$ 8.0). After incubation at $65^{\circ} \mathrm{C}$ for $10 \mathrm{~min}$, the suspended proteins were avoided by addition of equal volume of $\mathrm{PCl}$ (Phenol, Cholorofarm, Isoamyl alcohol in 25:24:1) followed by centrifugation (10000 rpm for $20 \mathrm{~min}$ ). The DNA from upper aqueous phase was precipitated by addition of two volumes of ice cold isopropanol preceded with $70 \%$ ethanol wash. The air dried pellet was dissolved in $50 \mu \mathrm{l}$ TE buffer, quantified using

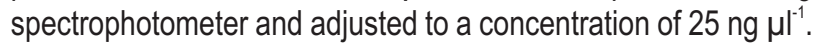
The residual RNA was eliminated by RNase treatment at $37^{\circ} \mathrm{C}$ for $30 \mathrm{~min}$.

PCR amplification : The details of primers used for amplification of gyrB, ERIC I, ERIC II, GTG and BOX-PCR is given in Table 1. The reaction mixture contained $50 \mathrm{ng}$ DNA, $300 \mu \mathrm{M}$ each dNTP, $1 \mathrm{X}$ reaction buffer, $0.2 \mu \mathrm{M}$ of each primer, $2.5 \mathrm{mM} \mathrm{MgCl}, 1.25 \mathrm{U}$ Taq DNA polymerase in a total volume of $20 \mu \mathrm{l}$. The gyrB fragment was amplified in a thermocycler (Biorad) programmed with an initial denaturation step at $94^{\circ} \mathrm{C}$ for $5 \mathrm{~min}$, followed by 35 cycles of amplification consisting initial denaturation at $94^{\circ} \mathrm{C}$ for $1 \mathrm{~min}$, annealing at $46^{\circ} \mathrm{C}$ for $1 \mathrm{~min}$, extension at $72^{\circ} \mathrm{C}$ for $1 \mathrm{~min}$. and a final extension step at $74^{\circ} \mathrm{C}$ for $10 \mathrm{~min}$. Whereas, the annealing temperatures for ERIC, GTG and BOX-PCR were $54^{\circ} \mathrm{C}, 42^{\circ} \mathrm{C}$ and $60^{\circ} \mathrm{C}$, respectively. Each cycle extension time was increased to 8 and 4 min for ERIC and GTG programs for better visualization of amplicons. A negative control without target DNA was also included in each experimental run.

Electrophoretic analysis: The amplicons and patterns were resolved in agarose (1\%) gel electrophoresis of $15 \mu \mathrm{l}$ of PCR product. The gels were run in TAE ( $40 \mathrm{mM}$ Tris-acetate, $1 \mathrm{mM}$ 
Table 1 : Details of the primers used in the study

\begin{tabular}{lll}
\hline Primer name and sequence & Amplicon pattern/gene & Annealing temperature $\left({ }^{\circ} \mathrm{C}\right)$ \\
\hline BCFW1 - 5'-GTTTCTGGTGGTTTACATGG-3' & gyrB (352 bp) & 46 \\
BCRW1 - 5'-CAACGTATGATTTAATTCCACC-3' & & \\
ERIC I - 5'ATGTAAGCTCCTGGGGATTCAC'3 & ERIC I & 54 \\
ERIC I - 5'ATGTAAGCTCCTGGGGATTCAC'3 & ERIC II & 54 \\
ERIC II - 5'AAGTAAGTGACTGGGGTGAGCG'3 & & \\
GTG - 5'GTGGTGGTGGTGGTGGTG'3 & GTG & 42 \\
BOX -5 'GATCGGCAAGGCGACGCTGAC'3 & BOX & 60 \\
\hline
\end{tabular}

Table 2 : Details of Bt isolates and GeneBank Accession numbers of the gyrB sequences

\begin{tabular}{lllll}
\hline S.No & Isolate & Collection site & Altitude $(\mathrm{m})$ & Accession number \\
\hline 1 & UKBt3 & Pantnagar & 344 & KX025078 \\
2 & UKBt5 & Tanakpur & 255 & KX025079 \\
3 & UKBt10 & Ramnagar & 345 & KX025080 \\
4 & UKBt15 & Darim & 1850 & KX025081 \\
5 & UKBt28 & Ladgav & 1518 & KX025082 \\
6 & UKBt33 & Dunagiri & 2400 & KX025083 \\
7 & UKBt36 & Gwaldam & 1960 & KX025084 \\
\hline
\end{tabular}

Table 3 : Patterns of nucleotide substitution (MCL estimate) in gyrB fragments of $B t$ isolates native to Uttarakhand Himalayas, India

\begin{tabular}{lllll}
\hline & A & T & G & C \\
\hline A & - & 12.26 & 7.05 & 1.18 \\
T & 18.39 & - & 1.24 & 8.77 \\
G & 18.39 & 2.16 & - & 8.77 \\
C & 2.48 & 12.26 & 7.05 & - \\
\hline
\end{tabular}

Only entries within a row should be compared. Substitutions are shown in bold and those of transversions are shown in italics

EDTA) buffer at $2 \mathrm{~V} / \mathrm{cm}$ for $5 \mathrm{hrs}$. After staining with ethidium bromide, bands were visualized using UV illumination and documented with an Alpha image analyzer (Bio-Rad).

Analysis of PCR patterns : Polymorphic bands from each PCR patterns were individually identified by their specific migration rates in the electrophoretic analyses by calculating molecular size of bands against 100 bp DNA ladder (Invitrogen, USA). Once bands were properly and distinctively identified, binary (0/1) matrices were constructed to compare the patterns. Jaccard's similarity coefficients were generated by the SIMQUAL subroutine from the NTSYS- pc 2.02 (Applied Biostatistics, Inc.) package. Cluster analysis along with their corresponding dendrograms were generated by the unweighted-pair group method using average linkages (UPGMA), with the SAHN and TREE subroutines from the NTSYS-pc package.

Sequencing and analysis of gyrB fragments : Based on UPGMA dendrogram and REP-PCR patterns seven major clusters were observed, based on which a total of seven $B t$ isolates (UKBt3, UKBt5, UKBt10, UKBt15, UKBt28, UKBt33 and UKBt36) were selected for sequencing of gyrB fragments. After amplification, the PCR products of gyrB fragments were purified using gel elution columns (Sigma) and sequenced from Scigenome labs, Kochi, India. Nucleotide sequences were aligned with the Clustal Omega (1.2.1) multiple sequence alignment (McWilliam et al., 2013) and molecular evolutionary analyses were performed using the software MEGA4 (Molecular Evolutionary Genetic Analysis version 4) (Tamura et al., 2007). The phylogenetic tree was constructed by the neighbor-joining method using the distance matrix from the alignment along with other published sequences of different Bt serovars (Accession numbers given in tree).

\section{Results and Discussion}

The AP-PCR electrophoretic patterns of Uttarakhand Bt isolates showing polymorphic bands is presented Fig. 1. They revealed a total of 32 polymorphic DNA fragments. The number of fragments using ERIC 1 primers varied from 1 to 9 per isolate, with a total of 10 electrophoretic patterns with the amplicons size ranging between 182 to 2178 bp (Fig. 1A). ERIC II primers generated 1 to 8 fragments per strain with a total of 12 electrophoretic patterns with fragments ranging from $378 \mathrm{bp}$ and 
2983 bp (Fig. 1B). BOX primer generated between 5 and 10 fragments and a total of 16 electrophoretic patterns with fragment sizes between 323 and 2011 bp (Fig. 1C). The GTG primers generated six electrophoretic patterns with fragment sizes ranging between 500 to 3025 bp (Fig. 1D). The ERIC and BOX-PCRs were the most informative. Predominant amplicons in ERIC 1, ERIC 2, BOX and GTG PCRs were found to be $600 \mathrm{bp}(71.1 \%), 572 \mathrm{bp}$ (77.7\%), $2000 \mathrm{bp}(37.8 \%)$ and $3025 \mathrm{bp}(91.1 \%)$ respectively.
In both the ERIC-PCRs, Bt isolates from most of the plain and low to mid hill regions (twenty seven isolates from UKBt1 to UKBt27, accounts to 60\%) showed similar amplicon patterns. Overall, most of hill isolates showed highly diverse and unique patterns among them. Amongst the plain and low to mid hill isolates, only two isolates (UKBt5 and UKBt6) showed unique amplicon patterns $(7.4 \%)$ which were isolated from Tanakpur and Raiwala, respectively. In addition, one isolate, UKBt10 showed
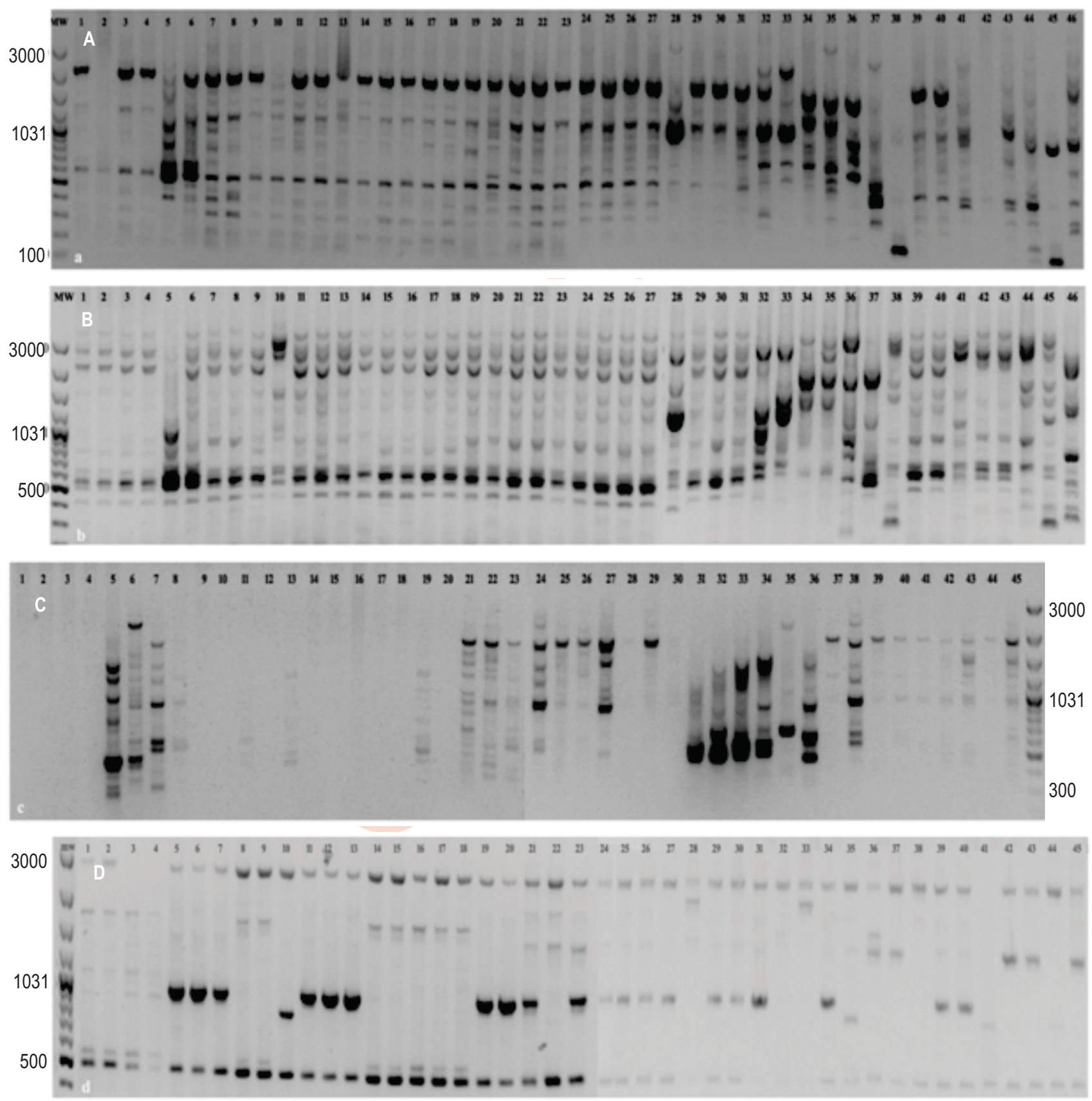

Fig. 1: AP-PCR patterns of $B$. thuringiensis isolates native to Uttarakhand Himalayas. (a) ERIC1; (b) ERIC2; (c) BOX and (d) GTG. The numbering of each lane indicates the Bt strain isolated from North Western Indian Himalayas 
little divergent pattern in ERIC2-PCR which was isolated from Bhowali. The remaining 24 amplicon patterns were similar and can be represented as single genotype. This altitudinal variation indicates the microclimate and ecosystem reliant evolution of the bacterium (Garcia et al., 2015; Zara et al., 2006; Vilas-Boas and Lemos, 2004). The soaring diversity observed here in the high hill isolates may be due to spatial separation of the strains generated by conquest of hills and mountains.

The BOX-PCR generated no amplicons in 16 isolates. This has been confirmed by permutations of primer and DNA concentrations. However, the isolates with amplicons showed unique patterns except UKBt39, UKBt40, UKBt41, UKBt42, UKBt43, UKBt44 and UKBt45 which showed similarity. All these isolates were collected from altitude range of 1890 to $2200 \mathrm{amsl}$ from Munsyari, Joshimath and Uttarakhasi areas. The same isolates showed little diversity in ERIC and GTG patterns which shows the resolving power of BOX-PCR. de Silva and Valicente (2013) also reported that BOX-PCR is most informative than ERIC. Taken as a whole, the AP-PCR patterns showed a substantial variation in Uttarakhand $B t$ isolates irrespective to the geographical variation indicating heterogeneous distribution with relative low relatedness (Garcia et al., 2015). Studies also reported the use of AP-PCR primers in phenotyping of $B t$ serotypes (Katara et al., 2012) and wild type collection from India (Katara et al., 2012), Russia (Boulygina et al., 2009), Mexico (Garcia et al., 2015) and Spodoptera frugiperda toxic collection from Embrapa (de Silva and Valicente, 2013). The dendrograms generated from all the three amplification patterns (Fig. 2) showed high phenotypic variability among the $45 \mathrm{Bt}$ isolates.

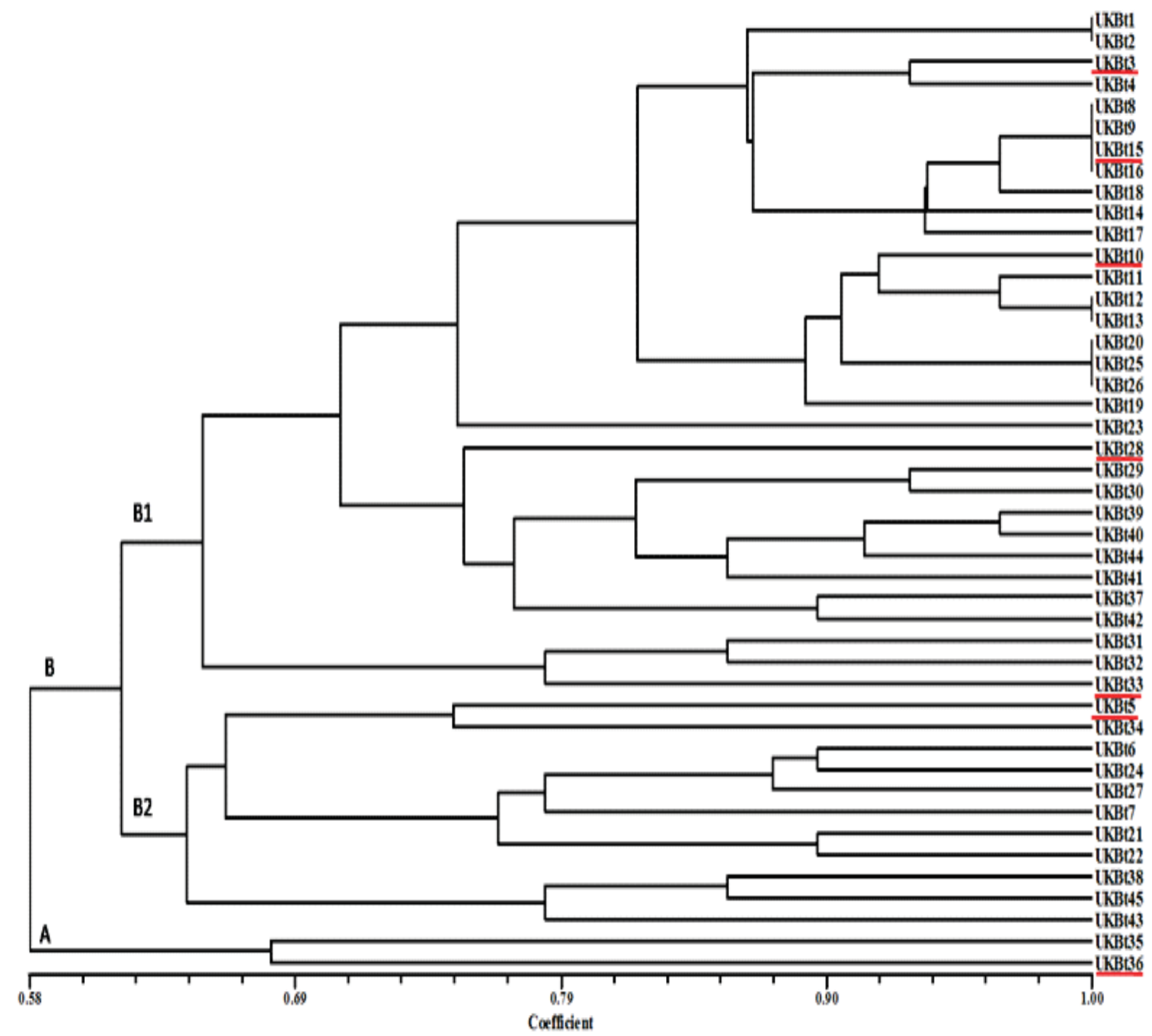

Fig. 2 : Dendrogram generated from AP-PCR patterns of 45 Bt isolates. The coefficient scale close to 1 indicates high similarity values. The isolates sequenced for gyrB were underline 


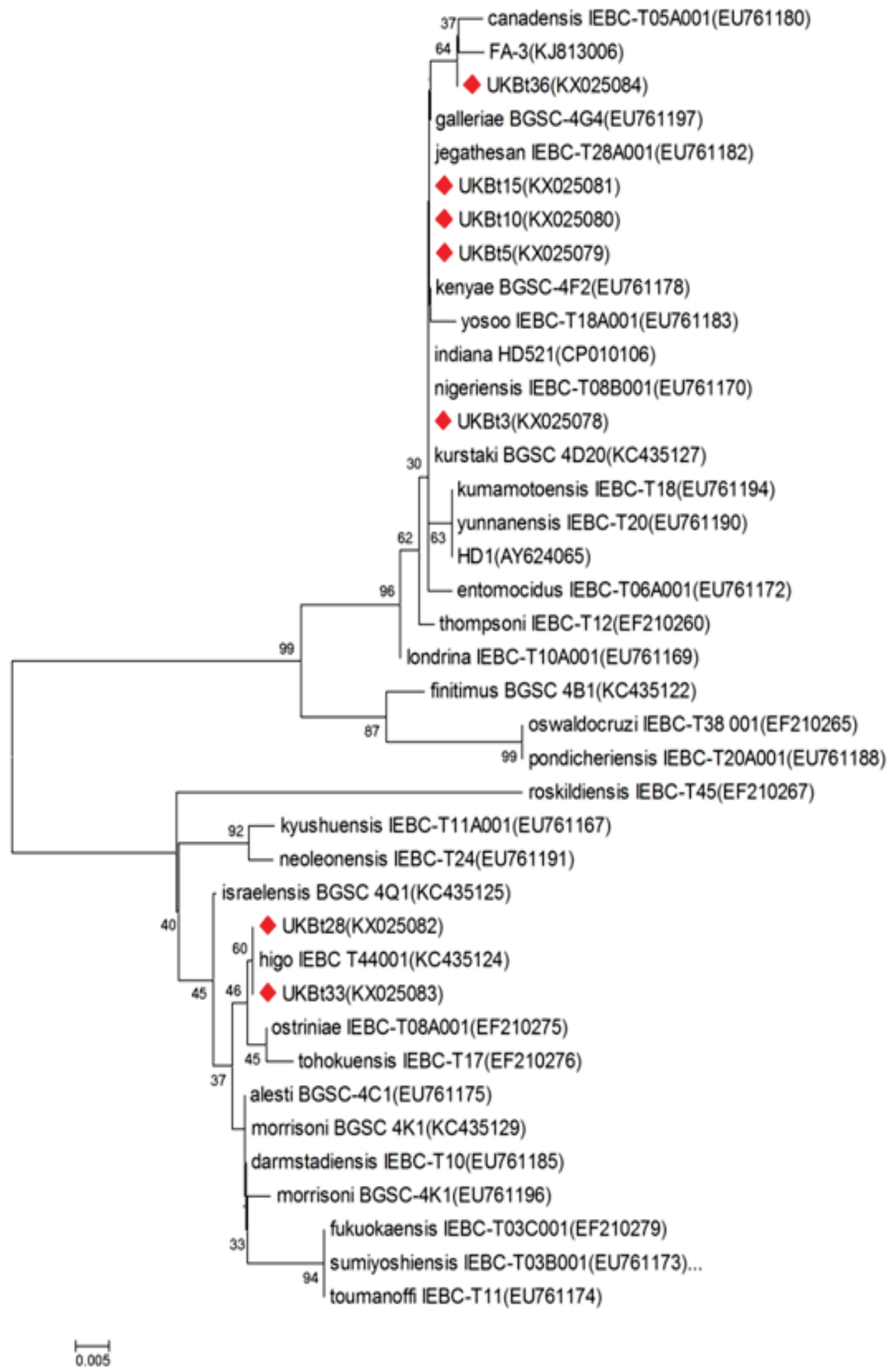

Fig. 3 : Phylogenetic tree based on the gyrB sequences of $B$. thuringiensis isolates native to Uttarakhand Himalayas, India in relation to different serovars constructed using neighbor-joining method. The genetic distances were computed by Kimura's model. The numbers at the nodes indicated percentages of occurrence in 1000 bootstrapped trees. Accession numbers were given in parenthesis. Boot strap values higer than 30 were indicated 
Even though there are two main clusters, the degree of similarity among strains between and within branches was low, as revealed by the multiple secondary branches and very dissimilar coefficient values. The two major groups, "A" and "B", were formed at a similarity level of 0.58 . The largest group "B " consists of 43 isolates covering both hill and plane isolates. However, the group "A" consists of only two isolates, UKBt35 and UKBt36 isolated from around $2000 \mathrm{~m}$ elevation. The largest cluster was further divided into two major branches (B1 and B2) at a similarity coefficient of 0.61 , which contained mixed elevation isolates. Interestingly, an observation on grouping of the isolates at high similarity values showed coexistence of sequentially collected samples which indicates genetic relatedness with respect to the geographical proximity that is at diminutive levels.

The gyrB sequences were submitted to NCBI Gene Bank nucleotide sequence databases (Table 2). Similarity search using the Blast programme (http://www.ncbi.nlm.nih.gov/Blast.cgi) showed close relatedness to gyrB sequences of different $B t$ strains and serovars. Moreover, a multiple sequence alignment of 285 bp showed a high similarity level with nucleotide substitutions viz., 23 single nucleotide polymorphisms and one pair base substitutions. These substitutions resulted in a neighbor joining phylogenetic tree with two distinct branches, the first one with UKBt28 and UKBt33 (the high hill isolates) with bootstrap consensus of 100 and the second one with remaining plane and mid hill isolates (Fig. 3). In this second clade, the isolate, UKBt36 was found to be distinct among the rest (UKBt5, UKBt3, UKBt10 and UKBt15). The tree also showed phylogenetic proximity of UKBt28 and UKBt33 with $B t$ serovar higo. The high hill isolate, UKBt36 was associated with Bt serovar canadensis. In addition, some serovars showed low indexes of statistical confidence indicating the taxonomic relatedness between the serovars. The nucleotide substitution patterns of gyrB sequences is presented in Table 3. The analysis showed an overall nucleotide composition of A, T, C and $\mathrm{G}$ as 39.6, 26.4, 15.2 and $18.9 \%$, respectively. The transition/transversion rate ratios for purines $\left(k_{1}\right)$ and pyrimidines $\left(k_{2}\right)$ were found to be 0.135 and 0.176 , respectively with overall transition/transversion bias of $0.046(R)$.

Single-strand conformation polymorphisms of the $16 \mathrm{~S}$ rDNA amplification products (a frequently used modern bacterial classifications and taxon identifications method) do not allow species discrimination within the Bacillus anthraciscereus-thuringiensis group (Borin et al., 1997). In this group, sequence analysis of a house keeping gene, gyr $B$ is found to be more highly differential than $16 \mathrm{~S}$ (La Duc et al., 2004; Huang, 1996). Studies have also reported the discriminatory power of this technique in native Bt collection (Awad et al., 2007; Punina et al., 2013), as well as serovars from the same $\mathrm{H}$-serotype and strains from same serovar (Soufiane and Cote, 2009). Awad et al. (2007) utilized the nucleotide substitution patterns by gyrB PCR-RE digestion using Sau3Al to distinguish between Bt strains. The originality of this study was the identification followed by confirmation of the exceptionality of native $B t$ strains based on REP-PCR patterns and gyrB sequences, respectively. Punina et al. (2013) reported an agreement between the AP-PCR and gyrB allocation of $B t$ isolates from Ukraine. The nucleotide conservation patterns and resulting phylogeny between the native isolates clearly differentiated isolates from plain or low hill areas (UKBt3, UKBt5, UKBt10 and 15) and high hill regions (UKBt28 and UKBt33) with an exception of UKBt36. This variability also suggests the microclimate dependent evolutionary and ecological relationships between the Btisolates.

In conclusion, the present study showed Bt collection from the Uttarakhand Himalayas represent a diverse members of $B t$ family. This uniqueness revealed from the AP-PCR and gyrB gene analysis may be the result of prevailing altitudinal variations within the mountain ranges that supported microclimate driven evolutionary discrepancies in Bt. Among the AP-PCR patterns, ERIC and GTG-PCR clearly indicated this ecosystem driven variability among the Bt isolates. Altitudinal dependent difference is clear in ERIC pattern whereas GTG patterns indicated variability between plane isolates. Overall, the study identified putative $B t$ isolates (UKBt3, UKBt35 and UKBt36), which are genetically divergent from the rest of the isolates. In view of this, further studies were planned to identify their uniqueness in bioactivity against insect pests, cry gene content, biotechnological applications etc.

\section{Acknowledgment}

The authors are grateful to the Director, ICAR-VPKAS, Almora for providing the facilities and financial support to undertake the investigations.

\section{References}

Akhurst, R.J., E.W. Lynes, Q.Y. Zhang, D.J. Cooper and D.E. Pinnock: A 16S rRNA gene oligonucleotide probe for identification of Bacillus thuringiensis isolates from sheep fleece. J. Invertebr. Pathol., 69, 24-31(1997).

Awad, M.K., I. Saadaoui, S. Rouis, S. Tounsi and S. Jaoua: Differentiation between Bacillus thuringiensis strains by gyrB PCR-Sau3Al fingerprinting. Mole. Biotechnol., 35, 171-178 (2007).

Borin, S., D. Daffonchio and C. Sorlini: Single strand conformation polymorphism analysis of PCR-tDNA fingerprinting to address the identification of Bacillus species. FEMS Microbiol. Letters, 157, 87-93 (1997).

Boulygina, E.S., A.N. Ignatov, S.V. Tsygankova, E.V. Korotkov and B.B. Kuznetsov: Interspecies relations between Bacillus thuringiensis strains studied by AP-PCR and sequence analysis of ribosomal operon regions. Microbiol., 78, 703-710 (2009).

Brousseau, R., A. Saint Orange, G. Pre Fontain, L. Masson and J. Cabana: Arbitrary primer polymerase chain reaction a powerful method to identify Bacillus thuringiensis serovar and strains. Appli. Environ. Microbiol., 59, 114-119 (1993).

Carozzi, N.B., V.C. Kramer, G.W. Warren, S. Evola and M.G. Koziel: Prediction of insecticidal activity of Bacillus thuringiensis strains by 
polymerase chain reaction product profiles. Appli. Environ. Microbiol., 57, 113057-113061 (1991).

Chandlera, D., G. Davidsona, W.P. Grantb, J. Greavesb and G.M. Tatchell: Microbial biopesticides for integrated crop management: An assessment of environmental and regulatory sustainability. Trends Food Sci. Technol., 19, 275-283 (2008).

de Silva, R.B. and F.H. Valicente: Molecular characterization of Bacillus thuringiensis using rep-PCR. SpringerPlus, 2, 641 (2013).

García, K., J.E. Ibarra, A. Bravo, J. Díaz, D. Gutiérrez, P.V. Torres and P.G. De Leon: Variability of Bacillus thuringiensis strains by ERICPCR and biofilm formation. Curr. Microbiol., 70, 10-18 (2015).

Huang, W.M.: Bacterial diversity based on type II DNA topoisomerase genes. Annu. Rev. Genet., 30, 79-107 (1996).

Joung, K.B. and J.C. Cote: A phylogenetic analysis of Bacillus thuringiensis serovars by RFLP-based ribotyping. J. Appli. Microbiol., 91, 279-289 (2001).

Katara, J., R. Deshmukh, N.K. Singh and S. Kaur: Molecular typing of native Bacillus thuringiensis isolates from diverse habitats in India using REP-PCR and ERIC-PCR analysis. J. General Appli. Microbiol., 58, 83-94 (2012).

Kumar, S., A. Chandra and K.C. Pandey: Bacillus thuringiensis (Bt) transgenic crop: An environment friendly insect-pest management strategy. J. Environ. Biol., 29, 641-653 (2008).

Kuo, W.S. and K.F. Chak: Identification of novel cry-type genes from Bacillus thuringiensis strains on the basis of restriction fragment length polymorphism of the PCR amplified DNA. Appli. Environ. Microbiol., 62, 1369-1377 (1996).

La Duc, M.T., M. Satomi, N. Agata and K. Venkateswaran: gyrB as a phylogenetic discriminator for members of the Bacillus anthracis-cereus-thuringiensis group. J. Microbiol. Methods, 56, 383-394 (2004).

Lima, A.S.G., A.M. Guidelli, I.L.D. Abreu and M.V.F. Lemos: Identification of new isolates of Bacillus thuringiensis using rep-PCR products and delta-endotoxin electron microscopy. Genetics Mole. Biol., 25, 225-229 (2002).

Malkawi, H.I., F. al-Momani, M.M. Meqdam, I. Saadoun and M.J. Mohammad: Detection of genetic polymorphism by RAPD-PCR among isolates of Bacillus thuringiensis. New Microbiol., 22, 241-247 (1999).

McWilliam, H., W. Li, M. Uludag, S. Squizzato, Y.M. Park, N. Buso, A.P. Cowley and R. Lopez: Analysis Tool Web Services from the EMBLEBI. NucleicAcids Res., doi:10.1093/nar/gkt376 (2013).

Porcar, M. and V. Juárez-Pérez: PCR-based identification of Bacillus thuringiensis pesticidal crystal genes. FEMS Microbiol. Reviews, 26, 419-432. DOI: 10.1111/j.1574-6976.2003.tb00624.x (2003).

Punina, N.V., V.S. Zotov, A.L. Parkhomenko, T.U. Parkhomenko and A.F. Topunov: Genetic diversity of Bacillus thuringiensis from different geo-ecological regions of Ukraine by analyzing the $16 \mathrm{~S}$ rRNA and gyrB genes and by AP-PCR and saAFLP. Acta Naturae, 5, 16 (2013).

Sambrook, J. and D. W. Russell: "Molecular Cloning: A Laboratory Manual. pp3.3.17 3.32(2001).

Sanahuja, G., R. Banakar, R.M. Twyman, T. Capell and P. Christou: Bacillus thuringiensis: A century of research, development and commercial applications. Plant Biotechnol. J., 9, 283-300 (2011).

Soufiane, B. and J.C. Côté: Discrimination among Bacillus thuringiensis $\mathrm{H}$ serotypes, serovars and strains based on $16 \mathrm{~S}$ rRNA, gyrB and aroE gene sequence analyses. Antonie Van Leeuwenhoek, 95, 33-45(2009).

Tamura, K., J. Dudley, M. Nei and S. Kumar: MEGA4: molecular evolutionary genetics analysis (MEGA) software version 4.0. Mole. Biol. Evol., 24, 1596-1599 (2007).

Vilas-Boas, G.T. and M. Lemos: Diversity of cry genes and genetic characterization of Bacillus thuringiensis isolated of Brazil. Canadian J. Microbiol., 50, 605-6013 (2004).

Yamada, S., E. Ohashi, N. Agata and K. Venkateswaran: Cloning and nucleotide sequence analysis of gyrB of $B$. cereus, $B$. thuringiensis, B. mycoides and $B$. anthracis and their application to the detection of $B$. cereus in rice. Appli. Environ. Microbiol., 65, 1483-1490 (1999).

Zara, G., S. Zara, N. P. Manglia, G. Garau, C. Pinna, G. Laddu and M. Badrani: PCR- based methods to discriminate Bacillus thuringiensis strains. Annals of Microbiol., 56, 71-76 (2006). 